\title{
Correction to: Sentiment Analysis on Substance Use Disorder (SUD) Tweets Before and During COVID-19 Pandemic
}

Avineet Kumar Singh and Dezhi Wu (D)

\author{
Correction to: \\ Chapter "Sentiment Analysis on Substance Use Disorder \\ (SUD) Tweets Before and During COVID-19 Pandemic" \\ in: C. Stephanidis et al. (Eds.): HCI International \\ 2021 - Posters, CCIS 1421, \\ https://doi.org/10.1007/978-3-030-78645-8_77
}

Acknowledgment section of the originally published version of chapter 77 was not complete. Additional acknowledgment and grant number were added in respective section. 